\title{
Domestizierung des Leviathan? Südostasiens Verfassungen und ASEAN's neues Homogenitätskriterium demokratischer Verfassungsstaatlichkeit
}

\author{
Von Jörg Menzel, Phnom Penh*
}

\section{Einleitung}

Seit Jahren macht der Begriff der Konstitutionalisierung die Runde. Auf nationaler Ebene ist von einer globalen Emergenz des Verfassungsstaates die Rede, und auf regionaler sowie internationaler Ebene wird der Terminus, häufig eher unpassend, zur Beschreibung aktueller Entwicklungen genutzt. Gerade für so genannte Entwicklungsländer gehört die Analyse ihrer Verfassungsstrukturen zunehmend zur Evaluierung ihres Entwicklungs- und Transformationspotentials ${ }^{1}$, womit einerseits der Beitrag von ,good governance“ zu ökonomischer und sozialer Entwicklung, andererseits aber auch der Eigenwert eines auf individuelle und politische Freiheit ausgerichteten Verfassungssystems in den Vordergrund rückt ${ }^{2}$.

$\mathrm{Zu}$ den bislang international selten analysierten Verfassungsregionen gehört Südostasien $^{3}$. Dies erscheint bedauerlich, weil sich die Region in den zurückliegenden zwanzig Jahren in vielfacher Hinsicht auch in verfassungsrechtlicher Hinsicht dynamisch entwickelt hat. Ende 2007 haben die zehn Mitglieder der südostasiatischen Staatengemeinschaft, allgemein bekannt als ASEAN, überdies eine Charter für die Gemeinschaft unterschrieben, die nach Ratifikation durch alle Mitgliedstaaten planmäßig vor Ende 2008 in Kraft treten

Jörg Menzel, Dr. jur., Privatdozent an der Universität Bonn, z.Zt. Rechtsberater des Senats in Kambodscha, Phnom Penh (CIM). Der vorliegende Beitrag beruht auf Vorträgen, die der Verfasser im Sommer 2008 an der Universität Bonn sowie vor dem Arbeitskreis für Überseeische Rechtsvergleichung in Lübeck gehalten hat. E-mail: joergmenzel@ gmx.net.

1 Vgl. etwa den „Transformation Index“ der Bertelsmann-Stiftung (www.bertelsmann-transformation-index.de). Zum Stellenwert des Verfassungsrechts in der Law and Development-Debatte Brun-Otto Bryde, Constitutional Law in "old" and "new" Law and Development, VRÜ 2008, S. 10 ff.; zur Bedeutung von Demokratie und Menschenrechten David M. Trubek, The „Rule of Law" in Development Assistance: Past, Present, and Future, in: ders./ Alvaro Santos (Hg.), The New Law and Economic Development. A Critical Appraisal, Cambridge 2006, S. 74 (84). Amartya Sen, Development as Freedom, New York 1999.

Vgl. generell Clauspeter Hill / Jörg Menzel (Hg.), Constitutionalism in Southeast Asia, Vol. I III, Singapore 2008 (publiziert von der Konrad Adenauer Stiftung), Vol. I: National Constitutions / ASEAN Charter; Vol. II: Reports on National Constitutions, Vol. III: Selected Issues (im Erscheinen). Vgl. daneben allgemein Graham Hassall / Cheryl Saunders, Asia-Pacific Constitutional Systems, Cambridge 2002; inzwischen weithin veraltert Lawrence W. Beer (ed.), Constitutional Systems in Late Twentieth Century Asia, Seattle and London 1992; knapp und instruktiv Kevin YL Tan, Comparative Constitutionalisms: The Making and Remaking of Constitutional Orders in Southeast Asia, Singapore Journal of International and Comparative Law 6 (2002), S. 1 ff. 
wird. In dieser Charter findet sich für jeden, der mit ASEAN, der Heterogenität seiner politischen Systeme sowie der strikten Tradition wechselseitiger Nichteinmischung ein wenig vertraut ist, Überraschendes: In Artikel 1, der von den Zielen der Gemeinschaft spricht, sowie Artikel 2, der gemeinsame Prinzipien beschreibt, verpflichten sich die ASEAN-Mitgliedstaaten unter anderem auf Demokratie, Menschenrechte, Rechtsstaatlichkeit, Verfassungsstaatlichkeit, good governance und den Respekt vor dem Völkerrecht ${ }^{4}$. Gleich zweifach finden wir hier Homogenitätsklauseln, die dem Artikel 6 des Europäischen Unionsvertrages nicht nachzustehen scheinen. Ist Südostasien also nunmehr ein konsolidierter Raum moderner Verfassungsstaatlichkeit?

Nicht so schnell, mag einwenden, wer ein wenig die Nachrichten aus Südostasien verfolgt. Ist nicht in Thailand jüngst wieder einmal geputscht worden und befindet sich das Land nicht nach wie vor in einer Verfassungskrise, wie schon die Philippinen seit Jahren? Sind nicht Brunei absolute Monarchie, Myanmar seit Jahrzehnten Militärdiktatur und Vietnam sowie Laos kommunistische Einparteiensysteme? Haben nicht Singapur und Malaysia langjährige Traditionen in der Beschränkung politischer Freiheit? Wird nicht in Südostasien auch programmatisch ständig von „Asian Values“ geredet, die im Gegensatz zu Demokratie- und Menschenrechtskonzepten im dekadenten Westen stehen sollen? Kann es sich bei den genannten Formulierungen in der ASEAN-Charter also nicht nur um Rhetorik eines zahnlosen und rechtlich ohnehin weithin bedeutungslosen Verbandes handeln?

Im Folgenden wird versucht, Entwicklung und Stand der Verfassungsentwicklung in Südostasien zu skizzieren, um dann auf die Bedeutung der ASEAN'ischen Homogenitätsklauseln zurückzukommen. Weil diese Ausführungen insgesamt elf Staaten umfassen, kann vieles nur angedeutet werden. Gezeigt werden soll aber, dass es so etwas wie eine Entwicklung zum Verfassungsstaat gibt und dass zumindest in der Tendenz Werte wie Demokratie und Grundrechte prominent vertreten sind auf dem Marktplatz der Ideen in Südostasien.

Article 1: "The Purposes of ASEAN are: ... 7. To strengthen democracy, enhance good governance and the rule of law, and to promote and protect human rights and fundamental freedoms, with due regard to rights and responsibilities of the Member States of ASEAN; ..." Article 2 (2): ASEAN and its Member States shall act in accordance with the following principles: ... h) adherence to the rule of law, good governance, the principles of democracy and democratic government; i) respect for fundamental freedoms, the promotion and protection of human rights, and the promotion of social justice; j) upholding the United Nations Charter and international law, including international humanitarian law, subscribed to by ASEAN Member States; ..." Die Charter findet sich auf der ASEAN-Website (www.aseansec.org) und ist abgedruckt in Hill/ Menzel, (Fn. 3) Vol. I, S. 385 ff. Sie tritt gemäß Artikel 47 (4) dreißig Tage nach Ratifizierung durch den letzten Mitgliedstaat in Kraft. Zur letzten Ratifizierung durch Indonesien am 21.10.2008 vgl. die Presseerklärung des ASEAN-Sekretariats vom gleichen Tag (www.aseansec. org). 


\section{Historische Perspektiven}

Zunächst sei ein kurzer Blick in die Geschichte erlaubt. Fast sprichwörtlich ist die Rede von der „orientalischen Despotie“, ein Konzept, das sich in der Antike bereits bei Aristoteles findet, später etwa bei Montesquieu und Hegel auftaucht, um im 20. Jahrhundert von Wittfogel für den Ost-West-Konflikt re-aktiviert zu werden ${ }^{5}$. Zwar beziehen sich die Vertreter der Theorie von der Orientalischen Despotie üblicherweise nicht auf Südost-Asien, aber auch in dieser alten Kulturregion wird von demokratischen Strukturen in der vorkolonialen Geschichte soweit ersichtlich nicht berichtet. Es fehlt scheinbar die Entsprechung zur athenischen Demokratie, die aber ja auch eher Ausnahme als Regel in der vormodernen Verfassungsgeschichte Europas war (und zudem ihrerseits frauendiskriminierende Sklavenhaltergesellschaft). In der Zeit des europäischen Mittelalters gibt es manche Parallele zu Südostasien. Jayavarman II eint im Jahr 802 das Reich der Khmer ${ }^{6}$, kurz nachdem Karl der Große sein Frankenreich in die Nachfolge des römischen Imperiums gestellt hatte. Das Reich der Khmer wächst über Jahrhunderte und seine Hauptstadt Angkor ist zu Beginn des 13. Jahrhunderts mit vielleicht einer Million Einwohnern die wohl bevölkerungsreichste Stadt der Welt. Angkors Könige sind absolut, auch wenn die Dynastien instabil bleiben ${ }^{7}$. Die präkoloniale Staatenwelt Angkors und Südostasiens im Allgemeinen ist keine solche rationaler Staatlichkeit in Weber'schen Kategorien, ebenso wenig wie die außerasiatische Welt jener Zeit ${ }^{8}$, auch wenn die archäologischen Hinterlassenschaften einen zum Teil hohen Grad an Organisation belegen ${ }^{9}$. Freiheitsrechtlich ist Asien dem Europa seiner Zeit bisweilen wohl auch voraus. Der indische Nobelpreisträger Amartya Sen hat darauf hingewiesen, dass sich Nachweise religiöser Freiheit in der Umbruchzeit von Mittelalter zu Neuzeit gerade in Asien finden - zu einer Zeit, in der in Europa Religionskriege und Inquisition toben ${ }^{10}$. Pauschalvergleiche verbieten sich allerdings quer durch die Geschichte, denn weder Europa noch Asien (oder auch nur Südostasien) waren oder sind einheitliche Räume.

Karl A. Wittfogel, Die orientalische Despotie. Eine vergleichende Untersuchung totaler Macht, Frankfurt a.M. u.a. 1977; für eine jüngere Kritik der umstrittenen Wittfogel-Thesen s. etwa Udo Witzens, Kritik der Thesen Karl A. Wittfogels über den „hydraulischen Despotismus“ mit besonderer Berücksichtigung des historischen singhalesischen Theravada-Buddhismus, Diss. Heidelberg 2000 (ebda. S. 58 umfängliche Nachweise zum "Propagandamythos" vom 'Bösen Asien"” bei Autoren wie Herodot, Aristoteles, Machiavelli, Montesquieu und anderen).

David Chandler, A History of Cambodia, 4. Auflage, Boulder 2008, S. 39 ff.

7 Zum “Angkorean Kingship" näher Chandler, (Fn. 6) S. 53 ff.

8 Treffend etwa Norman G. Owen u.a., The Emergence of Modern Southeast Asia, Singapur 2005, S. $53 \mathrm{f}$.

Zur Debatte um Angkor als "hydraulische Gesellschaft" im Sinne von Wittfogel (Fn. 5) zusammenfassend John Tully, A Short History of Cambodia, Chiang Mai 2006, S. $43 \mathrm{ff}$.

Amartya Sen, Human Rights and Asian Values, New York 1997. 
Mit Ausnahme Thailands gerät ganz Südostasien zeitweise unter koloniale Herrschaft. Die Zeitspanne des Kolonialismus variiert erheblich, in den Philippinen beginnt sie mit der Ankunft der Spanier im 16. Jahrhundert, in Indochina erst in der zweiten Hälfte des 19. Jahrhunderts. Ein breites Spektrum an Kolonialmächten ist vertreten mit Spanien, Portugal, Holland, England, Frankreich und schließlich auch den USA. Wie anderswo auch, prägt die Kolonialzeit Staatsbildung und Rechtsentwicklung in der Region mit bleibenden Wirkungen weit über ihr Ende hinaus ${ }^{11}$. Politisch werden die Inselarchipele Philippinen und Indonesien beispielsweise überhaupt erstmals zu einheitlichen Räumen. Rechtlich werden die Staaten der Region entweder vom Common-Law oder vom kontinental-europäischen Rechtskreis beeinflusst; noch heute werden sie zumeist als Mitglieder des einen oder anderen qualifiziert, auch wenn solche Zuordnungen aufgrund der Komplexität der Einflüsse zum Teil problematisch erscheinen ${ }^{12}$.

Dem Klischee asiatischer Unterwürfigkeit ${ }^{13}$ entspricht Südostasien auch insofern nicht, als koloniale Beherrschung mitnichten zur Selbstverständlichkeit wird. Der erste Präsident der Philippinen bringt den Freiheitswillen seines Landes auf den Punkt:

„I prefer a country run like hell by Filipinos to a country run like heaven by Americans. Because, however bad a Filipino government might be, we can always change it. "14

Die Philippinen versuchen bereits Ende des 19. Jahrhunderts, sich vom Kolonialismus zu befreien und verabschieden eine erste Unabhängigkeitsverfassung ${ }^{15}$. Der Selbstbefreiungs-

Vgl. Nicholas Tarling, Nations and States in Southeast Asia, Cambridge 1998; zur Rechtsentwicklung während der Kolonialzeit M.B. Hooker, A Concise Legal History of South-East Asia, Oxford 1978, S. 123 ff.

Die Rechtsordnungen Südostasiens lassen sich zwar zum Teil relativ klar zuordnen (Singapur etwa zum Common Law), zum Teil sind beide aber vermischt (Philippinen). Zum Teil gibt es starke Einflüsse sozialistischen Rechtsdenkens (Vietnam, Laos, nachwirkend auch in Kambodscha), religiösen Rechts (islamisches Recht in Indonesien, Malaysia, Brunei; s. auch M.B. Hooker, Islamic Law in Southeast Asia, Australian Journal of Asian Law 4 [2002], S. 213 ff.) oder indigener Rechtstraditionen (insb. Adat Law in Indonesien). Vgl. zur Unmöglichkeit der pauschalen Zuordnung der Rechtsordnungen Südostasiens und zur Notwendigkeit von Einzelfallbetrachtung auch Andrew Harding, Comparative Public Law: Some Lessons from South East Asia, in: ders./ Erin Örücu (Hg.), Comparative Law in the $21^{\text {st }}$ Century, London u.a. 2002, S. 249 (264 f.). ders., Global Doctrine and Local Knowledge: Law in South East Asia, ICLQ 51 (2002), S. 33 ff. Zu gemischten Systemen generell Jacques du Plessis, Comparative Law and the Study of Mixed Legal Systems, in: Mathias Reimann /Reinhard Zimmermann (Hg.), The Oxford Handbook of Comparative Law, Oxford 2006, S. 477 ff.

Dieses zum Konzept der asiatischen Despotie gehörende vermeintliche „Charaktermerkmal“ der Asiaten findet ironischerweise heute noch Anhängerschaft in der Region selbst, wo die Protagonisten der Asian-Values-Doktrin regelmäßig auf eine positivere Einstellung der Asiaten zur Autorität hinweisen. Die Existenz äußerst kraftvoller Demonstrationskulturen in Staaten wie Thailand, Indonesien, Philippinen, Süd-Korea oder Taiwan ist nur eines der Indizien, die der Grundannahme widerstreiten. 
versuch scheitert allerdings zunächst und die Kontrolle geht schlicht von Spanien auf die USA über. Der Freiheitswille aber bleibt in den Philippinen, und er keimt auch ansonsten in der Region. Japan sucht sich daher im Zweiten Weltkrieg als Befreier zu präsentieren, entlarvt sich aber allzu gleichzeitig als neue Beherrschermacht. Zwar gelingt es den europäischen Mächten nach Ende des Zweiten Weltkriegs überwiegend noch einmal kurzfristig, die Kontrolle über ihre südostasiatischen Kolonien wiederzuerlangen, regelmäßig aber nur noch, um dann bald unfreiwillig oder mehr oder weniger freiwillig den Weg in die Unabhängigkeit zu eröffnen ${ }^{16}$. Wenige Ausnahmen bestätigen die Regel: In Brunei entschließt sich der Sultan, bis 1984 unter dem Schutzschirm Großbritanniens zu bleiben (wohl nicht zuletzt, um innenpolitisch seine Macht zu sichern) ${ }^{17}$. Ost-Timors Unabhängigkeit steht mit dem Ende der portugiesischen Herrschaft ohnehin relativ spät im Jahr 1975 an, verschiebt sich aber aufgrund gewaltsamer Annexion durch Indonesien noch einmal um ein weiteres Vierteljahrhundert.

Mit der Unabhängigkeit kommen zunächst überall Verfassungen ${ }^{18}$. Kein Staat der Region folgt der britischen Tradition ungeschriebenen Verfassungsrechts oder verzichtet aus anderen Gründen auf die Verabschiedung eines geschriebenen Verfassungsdokuments. Bisweilen werden die Verfassungen in aller Eile verfasst, so insbesondere in Indonesien, wo die Unabhängigkeitsverfassung zunächst explizit nur Interimsverfassung sein soll ${ }^{19}$. In der Sache weisen diese Verfassungen Einflüsse von außerhalb auf. In Kambodscha etwa lehnt sich die Verfassung von 1947 in vielen Konzepten deutlich an die seinerzeit aktuelle französische Verfassung an. Großbritannien hat bei der Entwicklung einer Verfassung für Malaysia/Singapur später zwar keine „Mutterverfassung“ zu bieten, aber Verfassungen anderer Ex-Kolonien (insbesondere Indien) dienen hier ggf. als Vorlagen. Es nimmt außerdem soweit möglich prozedural Einfluss auf die Verfassungsentstehung durch Mitgestaltung sowie Partizipation im Beratungsprozess. „Abgeschrieben“ werden die Unabhängigkeitsverfassungen freilich regelmäßig nicht, und zum Teil finden sich auch signifikante

„Malolos-Verfassung“ (1899); abgedruckt in Rufus B. Rodriguez, Constitutionalism in the Philippines, Manila 1997, S. 117 ff.

Zur Dekolonialisierung in Südostasien etwa A.J. Stockwell, in: Nicholas Tarling (Hg.), The Cambridge History of Southeast Asia. Volume Four. From World War II to present, Cambridge 1999, S. $1 \mathrm{ff}$.

Vgl. Tsun Hang Tey, Brunei - Entrenching an Absolute Monarchy, in: Hill/Menzel, (Fn. 3), Vol. II, S. 7 (10 ff.).

Gute Übersicht zum Verfassungsentstehungsprozess bei Tan, (Fn. 3) S. 3 ff.

19

Indonesiens rudimentäre Eil-Verfassung von 1945 wird dementsprechend auch 1949 durch eine föderale Verfassung ersetzt, die ihrerseits bereits nach acht Monaten durch eine weitere - wieder einheitsstaatliche - Verfassung abgelöst wird, die besonders dezidiert Demokratie und Menschenrechte zum Inhalt hat. Diese Verfassung wird freilich 1959 wieder durch die Verfassung von 1945 ersetzt, die seither (reformiert 1999-2002) in Kraft ist. Vgl. den Überblick bei Denny Indrayana, Indonesia - In Search for a Democratic Constitution (1945-2008), in: Hill/Menzel, (Fn. 3) Vol. II, S. 95 (60 ff.). 
Anknüpfungen an eigene Traditionen ${ }^{20}$. Eine bemerkenswerte späte Ausnahme stellt insofern allerdings wieder die Verfassung Ost-Timors aus dem Jahr 2002 dar, die (sieht man von einigen Ergänzungen im Grundrechtsbereich ab) in weiten Teilen wörtlich aus Portugal übernommen ist ${ }^{21}$.

Zwar finden sich schon in den Unabhängigkeitsverfassungen wie gesagt durchaus nationale Besonderheiten, die in der jeweiligen Landesgeschichte begründet sind. Überwiegend beruhen aber jedenfalls die Verfassungen der ersten Dekolonialisierungswelle in bemerkenswertem Maße auf modernen Konzepten von Demokratie und Menschenrechten. Freiheit nicht nur nach außen, sondern auch im Inneren prägt den frühen Zeitgeist, wie etwa der Blick auf die Verfassungen in den Philippinen (1935), Thailand (1946), Vietnam (1946), Myanmar (1947), Kambodscha (1947), Laos (1947) und Indonesien (1950) zeigt. Allerdings halten diese Verfassungen in der Sache oft nicht lange, denn schnell verliert die Demokratie an Boden in der Region. „Guided Democracy“ ist das beschönigende Wort, mit dem sich junge Regime in Kambodscha (unter Sihanouk) oder Indonesien (unter Sukarno) alsbald beschreiben ${ }^{22}$. Das geteilte Vietnam der 1950er bis 1970er Jahre mit seinen linken und rechten Regimen symbolisiert den Verfall des demokratischen Gedankens vielleicht am deutlichsten. Die Idee des liberalen Verfassungsstaates bleibt einstweilen auf der Strecke, unterstützt auch dadurch, dass die Region zunehmend zum Schauplatz des Ost-West-Konflikts wird. Die Sowjetunion und China unterstützen sozialistische bzw. kommunistische Regime, und der Westen neigt zur Unterstützung autoritär militaristischer Regime, wenn sie nur ,anti-kommunistisch“ sind. Malaysia und Singapur etablieren mit der Unabhängigkeit tendenziell Verfassungssysteme, die stark von ,,public order“ und der Beschränkung politischer Freiheiten geprägt sind. In zahlreichen Staaten der Region sind Verfassungen über Jahrzehnte jedenfalls keine effektiven Gewährleistungen für Demokratie, Menschenrechte oder substanzielle Rechtsstaatlichkeit. In einigen Staaten geben sich autoritäre Regime über missbräuchliche Verfassungsänderungen bzw. Notstandsgesetzgebung einen Anstrich der Legalität, wie etwa im Kambodscha unter Sihanouk oder den Philippinen unter Marcos, in anderen sind Verfassungen praktisch bedeutungslose Staatsrhetorik, wie in den kommunis-

Hierzu für Malaysia Abdul Aziz, Bari, The Indigenous Roots of the Malaysian Constitution - The Provisions and the Implications, unveröffentlichtes Manuskript für die $5^{\text {th }}$ Asian Law Institute Conference, Singapur 2008; ders., British Westminster System in Asia - The Malaysian Variation, US-China Law Review 4 (2007), S. 1 ff.

Zur Verfassunggebung in Ost-Timor näher Christian Roschmann, East Timor (Timor-Leste) Constitutional Framework for a Country in the Making, in: Hill/Menzel, (Fn. 3) Vol. II, S. 69 (75 ff.); Hillary Charlesworth, The Constitution of East Timor, May 20, 2002, ICON 1 (2003), S. 325 ff.

Sihanouk sichert seine Position u.a. über mehrere signifikante und in problematischen Verfahren beschlossene Änderungen der Verfassung von 1947 ab, während Sukarno sich 1959 der demokratisch-rechtsstaatlichen Verfassung von 1950 durch Rückkehr zur Eil-Verfassung von 1945 entledigt. 
tischen Regimen Indochinas. „Relevant“ als Steuerungselement für Politik und Recht ist die Verfassung hier wie dort nicht oder nur marginal.

Eine neue Phase der Verfassungsstaatlichkeit beginnt in den späten 1980er Jahren, bemerkenswerterweise weithin synchron mit der Stärkung der verfassungsstaatlichen Idee im Bereich des ehemaligen Ostblocks und anderen Teilen der Welt ${ }^{23}$. Den Anfang machen erneut die Philippinen mit dem Sturz Marcos', der in der neuen demokratischen Verfassung von 1987 mündet. Ende der 1980er Jahre beginnen in Kambodscha und Vietnam Reformprozesse, die ebenfalls mit neuen Verfassungen einhergehen, während man in Laos 1991 überhaupt erstmals seit 1975 wieder eine Verfassung in Kraft setzt. Thailand gibt sich nach intensiver Vorbereitung 1997 eine zunächst gepriesene und auf Demokratie, Grundrechte sowie Rechtsstaat gegründete Verfassung ${ }^{24}$. In Indonesien schließt sich an den Sturz Suhartos 1998 ein gestufter Reformprozess an, der im Wege von vier Verfassungsreformen in der Sache eine neue Verfassung hervorbringt und Indonesien zur Demokratie macht ${ }^{25}$. Die Verabschiedung der Verfassung Ost-Timors (2002) sowie jedenfalls formal signifikante Verfassungsreformen in Vietnam (2001) und Laos (2003) sind weitere Entwicklungsschritte im Konstitutionalisierungsprozess der Region.

Neben den positiven Entwicklungsschritten stehen freilich auch negative Entwicklungen bzw. fortdauernde Probleme. Dass es auch seit den späten 1980er Jahren nicht nur positive Entwicklungen gegeben hat, sondern auch problematische Entwicklungen und Rückschläge zu verbuchen sind, liegt auf der Hand. Thailands Militärcoup in 2006 beendete die Illusion, das Land sei mit der Verfassung von 1997 seinem scheinbar ewigen Zirkel aus Verfassungsgebung und Militärcoups entronnen. In den Philippinen scheitern Militärcoups zwar regelmäßig, gleichwohl befindet sich auch dieses Land seit Jahren gewissermaßen in einer Dauerverfassungskrise. In Brunei gab es 2004 eine Verfassungsreform, die das Prinzip der absoluten Monarchie auch formal zementierte, ${ }^{26}$ und in Myan-

Bruce Ackermann, The Rise of World Constitutionalism, Virginia Law Review 83 (1996), S. 83 ff.

Andrew Harding, May there be Virtue; ,New Asian Constitutionalism“ in Thailand, in: Australian Journal of Asian Law 3 (2001), S. 236 ff.

Die ausführlichste Untersuchung ist Denny Indrayana, Indonesian Constitutional Reform 19992002. An Evaluation of Constitution-Making in Transition, Diss. Melbourne 2005 (bislang unveröffentlicht); s. auch Andreas Ufen, Die Verfassungsreformen in Indonesien, VRÜ 2003, S. 225 ff.; Tim Lindsey, Constitutional Reform in Indonesia: Muddling Towards Democracy, in: ders. (Hg.), Indonesia. Law and Society, 2. Auflage Annandale 2008, S. 23 ff.; Sebastian Benesch, The Indonesian Constitution: Historical Developments and an Evaluation of Recent Constitutional Amendments, in: Bob S. Hadiwinata / Christoph Schuck (Hg.), Democracy in Indonesia. The Challenge of Consolidation, Baden-Baden 2007, S. $177 \mathrm{ff}$. 
mar inszenierten die Regierenden ein Referendum über eine Verfassung, die die militärische Kontrolle der Politik auch für die Zukunft absichern soll ${ }^{27}$.

\section{Verfassungsstaat}

Fragt man, die historische Entwicklung und den aktuellen Stand betrachtend, nach dem derzeitigen Stand der Verfassungsstaatlichkeit, mag man zunächst bezweifeln, dass sich so viel Veränderung, wie sie sich in Südostasien zuträgt, überhaupt mit der Idee des Verfassungsstaates vereinbaren lässt. Verfassungen, so wird häufig kolportiert, sollten auf Beständigkeit angelegt sein. Sie sollten nicht beständig ersetzt oder geändert werden. Gegen die Existenz einer verfassungsstaatlichen Kultur in Südostasien wird daher geltend gemacht, dass hier wenig Kontinuität herrsche ${ }^{28}$. Insofern ist aber zunächst festzuhalten, dass häufige Verfassungsänderungen auch anderswo vorkommen ${ }^{29}$. Mit Blick auf Südostasien relativiert sich im Übrigen bei näherer Betrachtung der Eindruck der Kurzlebigkeit von Verfassungstexten. Natürlich ist da Thailand mit seinen inzwischen 18 Verfassungen seit $1932^{30}$, aber die Zahl täuscht schon darüber hinweg, dass viel aufeinander aufbaut, es insofern durchaus eine kontinuierliche Entwicklung verfassungsrechtlicher Konzepte gibt, die von der Lebensdauer der jeweils konkreten Verfassungen unabhängig ist. Im Übrigen ist Thailand mit seiner Tradition des periodischen Militärcoups eben auch einzigartig in dieser Hinsicht (so wie Großbritannien in Europa einzigartig dadurch ist, dass es überhaupt keine geschriebene Verfassung hat). Indonesien hat seine Verfassung von 1946 zwischen 1999 und 2002 systematisch modernisiert, nicht allzu viel an Reform in über einem halben Jahrhundert, das mindestens zwei fundamentale politische Systemwechsel umspannt. Die Philippinen leben seit 1987 unter einer textlich unveränderten Verfassung. Singapur und Malaysia haben keine fundamentalen Wechsel, aber schon die Technizität dieser Verfassungen impliziert ständigen Anpassungsbedarf. Es mag hier auch eine britische Tradition nachwirken, die auf der Idee der „Supremacy of Parliament“ gegründet ist, nicht aber derjenigen einer dem Alltagsdiskurs enthobenen Verfassung, die eben kein Produkt alltäglicher Gesetzgebungsakte sein sollte.

Myint Zan, Myanmar (Burma) - From Parliamentary System to Constitutionless and Constitutionalized One-Party and Military Rule, in: Hill/Menzel, (Fn. 3) Vol. II, S. 189 ff.

Vgl. etwa Jürgen Ruland, Politische Systeme in Südostasien, München 1998, S. 50 ff.

In Indien witzelt man gar über die Anzahl der Verfassungsänderungen: Dort ging, so wird erzählt, ein Mann in ein Buchladen und fragte nach einer Ausgabe der Verfassung. ,Sorry Sir, we do not sell periodicals“, wurde ihm geantwortet. Zum Grundgesetz gibt es, auch hieran sei erinnert, seit 1949 über fünfzig Änderungsgesetze, im Schnitt fast jedes Jahr eins.

30

Für eine Auflistung s. Chaowana Traimas \& Jochen Hoerth, Thailand - Another New Constitution as a Way Out of the Vicious Cycle?, in: Hill/Menzel, (Fn. 3) Vol. II, S. 299 (309 ff.). Vgl. zur Einbettung der jüngsten Verfassung in die thailändische Verfassungsgeschichte auch Same Varayudej, Constitutionalism and the Rule of Law in Thailand, Konferenzmanuskript, Singapur 2008. 
Die Verfassunggebungs- und Verfassungsänderungsverfahren in Südostasien sind ebenso variantenreich wie anderswo ${ }^{31}$. Zum Teil entscheiden spezielle verfassunggebende Versammlungen, zum Teil reguläre Parlamente. Gerade in der Gründungsphase gab es häufig vorbereitende Kommissionen. In manchen Fällen sind die Verfassungsberatungen weithin transparent ${ }^{32}$ und begleitet von intensiver öffentlicher Diskussion, in anderen Fällen sind sie geheimnisvoll. Bisweilen gibt es Referenden. Letztere sind bemerkenswerterweise gerade dann häufig zu beobachten, wenn die demokratische Legitimität des Gesamtverfahrens besonders zweifelhaft ist, so zuletzt wieder in Thailand 2007 und Myanmar 2008. Thailands neue Verfassung ist überhaupt weniger wegen der Textsubstanz (die im Wesentlichen eine Weiterentwicklung der Verfassung von 1997 ist) als wegen des Verfahrens ihrer Verabschiedung zu kritisieren. Während die Regierung erhebliche Budgetmittel zur Werbung für den Entwurf zur Verfügung stellte, war Kritik am Entwurf offiziell unter Strafe gestellt und politischen Parteien die Werbung für ein „no-Vote“ untersagt ${ }^{33}$. Myanmar war jüngst allerdings in der Lage, ein noch absurderes Referendum zu initiieren ${ }^{34}$.

Der Vorrang der Verfassung ist in den Verfassungstexten Südostasiens heute regelmäBig ausdrücklich klargestellt. Ihre praktische Relevanz variiert gleichwohl erheblich. Während die Verfassung von Laos nach wie vor als eher narrativ zu qualifizieren ist ${ }^{35}$, haben die Verfassungen der Philippinen und Indonesiens im praktischen Staatsleben eine erhebliche alltägliche Relevanz. Von kaum zu überschätzender Bedeutung ist in diesem Zusammenhang, ob und wie Verfassungen durch Gerichte aktualisiert und interpretiert werden. Weltweit wurden in den zurückliegenden Jahrzehnten Verfassungsgerichte gerade in den zurückliegenden zwei Jahrzehnten massenhaft gegründet und auch in Staaten des CommonLaw-Rechtskreises kontrollieren Supreme Courts in zunehmendem Umfang Staatshandeln (einschließlich Gesetzgebung) am Maßstab der Verfassung. Dieser Trend lässt sich auch in Asien $^{36}$ sowie spezieller Südostasien feststellen. Verfassungsgerichte wurden 1997 in Thailand $^{37}$ und 2003 in Indonesien ${ }^{38}$ etabliert. Insbesondere letzteres hat sich innerhalb

31

32

33

34

35

36

37

Für kursorische Vergleichsperspektiven auch Hassall/Saunders, (Fn. 3) S. 54 ff. sowie Tan, (Fn. $3)$.

Für die aktuelle Verfassung der Philippinen von 1986 gibt es etwa einen interessanten Band zu den Verfassungsberatungen: Joacquin G. Bernas (Hg.), The Intent of the 1986 Constitution Writers, Manila 1995 (gewissermaßen ein Pendant zu JÖR N.F. 1 für das Grundgesetz).

Chaowana Traimas /Jochen Hoerth, in: Hill/Menzel, (Fn. 3) Vol. II, S. 302.

Für eine ausführliche Dokumentation s. Human Rights Watch, Vote to Nowhere. The May 2008 Constitutional Referendum in Burma, May 2008 (abrufbar unter www.hrw.org).

Vgl. auch Gerald Leather, Laos - A Constitution in Search of Constitutionalism, in: Hill/Menzel, (Fn. 3) Vol. II, S. 123 (S. 158 f.); zur Verfassung von 1991 auch Martin Stuart Fox, The Constitution of the Lao People's Democratic Republic, Review of Socialist Law 17 (1991), S. 299 ff.

Hierzu insbesondere auch Tom Ginsburg, Judicial Review in New Democracies. Constitutional Courts in Asian Cases, Cambridge 2003.

Zum thailändischen Verfassungsgericht s. Andrew Harding, A Turbulent Innovation: The Constitutional Court of Thailand, 1998-2006, unpublished working paper, Melbourne 2007. 
kürzester Zeit eine erhebliche Reputation erarbeitet. Es hat eine Reihe politisch sensitiver Gesetze verworfen und hat (im Gegensatz zur sonstigen Justiz in Indonesien) bislang den Ruf weitgehender Korruptionsfreiheit. In Thailand wurde das Verfassungsgericht mit der neuen Verfassung von 2007 erstmals in Südostasien mit einer beschränkten Zuständigkeit für Verfassungsbeschwerden ausgestattet ${ }^{39}$. Ein Verfassungsrat, in der Sache eine Mischung aus französischem Conseil Constitutionel und deutschem Verfassungsgericht, besteht seit 1998 in Kambodscha, versucht bislang aber so unauffällig wie möglich zu bleiben $^{40}$. In den Common Law Staaten der Region übt insbesondere der Supreme Court der Philippinen seine Befugnis zur Verfassungskontrolle auch gegenüber Regierungsakten durchaus selbstbewusst aus ${ }^{41}$, während umgekehrt der Supreme Court in Singapur nach verbreiteter Auffassung in ,politischen“ Verfahren bislang nur wenig zur Kontrolle von Legislative und Exekutive beigetragen hat ${ }^{42}$ und sein Pendant in Malaysia zudem auch erheblichen direkten Eingriffen in seine Unabhängigkeit ausgesetzt war ${ }^{43}$. Bemerkenswert erscheint wiederum, dass man selbst in Vietnam inzwischen über Reformschritte in Richtung juristischer Verfassungskontrolle nachdenkt, wobei man sich dort selbst offenbar noch nicht sicher ist, ob und wie sich ein solches Konzept mit der traditionellen Führungsrolle der Kommunistischen Partei verträgt ${ }^{44}$.

Mit Blick auf die allgemeine Natur südostasiatischer Verfassungsstaatlichkeit unvermeidbar ist noch die Frage nach dem Umgang mit Notstandssituationen ${ }^{45}$. Der Ausnahme-

Zur Entstehung des indonesischen Verfassungsgerichts Petra Stockmann, The New Indonesian Constitutional Court, Jakarta 2007.

Artikel 213 Verfassung Thailands. Die Bestimmung erfasst nur die Überprüfung der Grundrechtswidrigkeit von Gesetzen, nicht exekutiven Staatshandelns.

Hierzu Jörg Menzel, Cambodia - From Civil War to a Constitution to Constitutionalism?, in: Hill/Menzel, (Fn. 3) Vol. II, S. 39 (57 ff.).

Knappe Übersicht bei Harry Roque, in: Hill/Menzel, (Fn. 3) Vol. II, S. 213 (238 ff.).

Kevin Y.L. Tan, An Introduction to Singapore's Constitution, Singapur 2005, S. 138, fast den Stand der Dinge wie folgt zusammen: "The Courts are ... extremely practical in orientation and are prepared to give administrative authorities a wide berth. In the areas of public order and national security, the courts are especially sympathetic to executive and national concerns." S. auch Li-ann Thio, Singapore - The Indigenisation of a Westminster Import, in: Hill/Menzel, (Fn. 3) Vol. II, S. 249 (282 ff.).

C.L. Lim, Malaysia - (1957-2007) Fifty Years, Fifty Amendments and Four Principal Developments, in: Hill/Menzel, (Fn. 3) Vol. II, S. 161 (182 ff.); instruktiv auch Khoo Boo Teik, Between Law and Politics. The Malaysian judiciary since independence, in: Kanishka Jayasuriya ( $\mathrm{Hg}$.), Law, Capitalism and Power in Asia, London \& New York 1999, S. 205 ff.

Vgl. Mark Sidel, Law and Society in Vietnam, Cambridge 2008, S. 50 ff.; Clauspeter Hill, Vietnam - Constitutional Development in a Re-United Country, in: Hill/Menzel, (Fn. 3) Vol. II, S. 327 (349 ff.). Constitutionalism?, sowie Kevin Y.L. Tan, From Myanmar to Manila: A Brief Study of Emer- 
zustand ist, so ist man versucht zuzuspitzen, in Teilen Südostasiens fast Normalzustand. In Brunei, das faktisch wohl einer der ruhigsten Staaten der Welt ist (sieht man vom allgegenwärtigen Gesang aus den Moscheen $a b$ ), wird ein lange vor Staatsgründung ausgerufener Ausnahmezustand routinemäßig alle zwei Jahre verlängert ${ }^{46}$. Die Diktatoren Marcos und Suharto stabilisierten ihre Macht über Jahrzehnte auf der Grundlage (antikommunistischer) Ausnahmebestimmungen und repressiver Staatsgewalt und auch Singapur $^{47}$ sowie Malaysia ${ }^{48}$ definieren sich seit Jahrzehnten als Staaten, in denen alle möglichen Sondersituationen von kommunistischer Aggression bis zu ethnischer Vielfalt besondere Gesetze und Maßnahmen erforderlich machen sollen. Natürlich hat in jüngerer Zeit zudem „September 11“ seine Spuren hinterlassen, denn wie anderswo hat man auch in Südostasien die Gelegenheit genutzt, Gefahrenabwehrrecht über den engeren Gegenstand der Terrorismusbekämpfung hinaus zu verschärfen ${ }^{49}$.

Gleichzeitig deutet sich in jüngerer Zeit aber an, dass auch in Südostasien der Rekurs auf Notsituationen nicht mehr unhinterfragt bleibt. Immerhin wurde wie erwähnt in Thailand im Zuge des Militärcoups 2006 das Verfassungsgericht gerade deshalb unmittelbar aufgelöst, um sicherzustellen, dass der Coup nicht gerichtlich in Frage gestellt würde ${ }^{50}$. In den Philippinen erklärte der Supreme Court in 2006 einen von Präsidentin Arroyo erklärten nationalen Notstand für rechtswidrig ${ }^{51}$. In Indonesien erklärte das noch junge Verfassungsgericht einschlägige Anti-Terrorismus-Gesetzgebung im Nachklang der Bali-Bombings wegen Verstoßes gegen das Rückwirkungsverbot für verfassungswidrig ${ }^{52}$; zuvor hatte das Gericht bereits ein Wahlverbot für ehemalige Mitglieder der kommunistischen Partei nach

gency Powers in Southeast Asia (bislang unveröffentlichte Konferenzmanuskripte, Singapur 2008).

Tsun Hang Tey, in: Hill/Menzel, (Fn. 3) Vol. II, S. 7 (33 f.).

In Singapur erlauben Artikel 149 und 150 die weitgehende Nichtbeachtung von normalen verfassungsrechtlichen Garantien im Fall von Anti-Subversions- und Notstandsgesetzgebung; hierzu etwa Tan, An Introduction to Singapore's Constitution, Singapur 2005, S. 150 f.

Vgl. für einen viel diskutierten Problemkreis Human Rights Watch, Convicted Before Trial. Indefinite Detention Under Malaysia's Emergency Ordinance, August 2008 (www.hrw.org).

Vgl. beispielsweise Salvador T. Carlota, The Philippine Anti-Terror Law: Constitutional Issues and Perspectives, in: The Asian Forum on Constitutional Law 2007: Constitutional Changes and Asian Constitutionalism in the $21^{\text {st }}$ Century, Nagoya 2008, S. $35 \mathrm{ff}$.

So etwas ist auch in der asiatisch-pazifischen Region nicht mehr undenkbar. In benachbarten Südpazifik hatte der fidschianische Court of Appeal 2001 einen Militärcoup für gescheitert erklärt. Hierzu eingehend Jörg Menzel, Revolution vor Gericht: Fidschis Court of Appeal erklärt Verfassungsputsch für gescheitert, VRÜ 2001, S. $148 \mathrm{ff}$. David vs. Arroyo, G.R. No. 171396 (3. Mai 2006).

Hierzu Simon Butt \& David Hansell, The Mayskur Abdul Kadir Case: Indonesian Constitutional Court Decision No 013, Australian Journal of Asian Law 6 (2004), S. 176 ff. 
Jahrzehnten für verfassungswidrig erklärt ${ }^{53}$. Der Versuch, ein neues Notstandsgesetz durchzusetzen, war in Indonesien bereits 1999 aufgrund öffentlichen Widerstands im letzten Moment gescheitert. Das Gesetz war schon vom Parlament bestätigt, als der Präsident sich im Angesicht von Demonstrationen entschloss, es nicht zu unterschreiben. Die Berufung auf „Emergency“ ist auch in Südostasien, das deutet sich jedenfalls an, kein Wundertrank mehr für einen urgewaltigen Leviathan.

\section{Demokratie}

Wenden wir uns nun den Sachthemen des Verfassungsrechts und hier zunächst der Demokratiefrage $\mathrm{zu}^{54}$. Will man sich der Frage eines möglichen besonderen Demokratieverständnisses, wie es im formalen Verfassungsrecht seinen Ausdruck findet, annähern, wird man zunächst die „,vollsäftige“ Monarchie Bruneis (wie Otto Mayer gesagt hätte) und Militärdiktatur Myanmars ausblenden müssen. Beide Staaten werden auch in der Region selbst klar als Anomalien angesehen. Dabei bleibt das kleine, reiche und unauffällige Brunei international weithin außerhalb der Wahrnehmung, während die Anomalie in Myanmar auch in ASEAN ein hochgradiges Politikum ist ${ }^{55}$. Vietnam und Laos verstehen sich selbst offiziell natürlich als Demokratien, aber es handelt sich eben nach wie vor um, wenn auch wirtschaftsliberalisierte, kommunistische "Volksdemokratien". Kambodscha ist formal liberale und pluralistische Demokratie westlichen Musters, aber in der Sache autoritäres „strongmanship“ “56. Singapur ist seit Jahrzehnten de-facto-Einparteienstaat mit restriktiven Strukturen im Bereich politischer Freiheiten und einem auf Machtstabilisierung zugeschnittenen Wahlsystem ${ }^{57}$. Das Konzept echter Wettbewerbs-Demokratie findet sich derzeit

Für eine Übersicht der wichtigsten Entscheidungen des neuen Verfassungsgerichts s. auch Denny Indrayana, in: Hill/Menzel, (Fn. 3) Vol. III sowie Stockmann, Fn. 38, S. 44 ff.

Für eine solide politikwissenschaftliche Darstellung der südostasiatischen Systeme s. Damien Kingsbury, South-East Asia. A Political Profile, 2. Auflage, Oxford 2005; Berichte zu aktuellen Entwicklungen in den einzelen Staaten finden sich in dem jährlich vom „Institute of Southeast Asian Affairs“ (ISEAS) in Singapur herausgegebenen ,Southeast Asian Affairs“.

Vgl. nur C.L. Lim: From Constructive Engagement to Collective Revulsion, The Myanmar Precedent of 2007, unpubliziertes Manuskript (zur Publikation vorgesehen im Singapore Law Review). McCargo, Cambodia. Getting Away with Authoritarianism?, Journal of Democracy 16 (2005), S. 98 ff.; eingehend (mit Qualifizierung Kambodschas als „defekte Demokratie“ Christina Motejl, Transformation und Demokratisierung in Kambodscha - Die Rolle der politischen Institutionen, Bonn 2007 (unveröffentlichte Magisterarbeit).

57 Vgl. hierzu näher Tsun Hang Tey, Singapore's Electoral System: Government by the People?, in: Hill/Menzel, (Fn. 3) Vol. III; Li-ann Thio, The Right to Political Participation in Singapore: Tailor-Making a Westminster-Modelled Constitution to Fit the Imperatives of 'Asian' Democracy, Singapore Journal of International and Comparative Law 6 (2002), S. $181 \mathrm{ff}$. 
insbesondere in Indonesien, den Philippinen und Thailand ${ }^{58}$, sowie in zunehmendem Umfang auch in Malaysia.

Bemüht man die Kategorien Max Webers, ${ }^{59}$ gibt es eher legalistisch-bürokratische, charismatische und traditionale Herrschaft. Singapur ist im Grunde ein Musterbeispiel legalistisch bürokratischer Herrschaft. Es hat den mit ca. 2 Mio. US Dollar per anno offiziell bestbezahlten Regierungschef der Welt, aber sein Erfolg liegt nicht im Charisma begründet, sondern im ökonomischen Leistungsnachweis seines eher wie ein internationales Großunternehmen denn ein asiatischer Staat geführten Gemeinwesens ${ }^{60}$. Als Beispiel traditionaler Herrschaft kann insbesondere Brunei gelten. In Thailand ist der König eine die häufig wechselnden Verfassungsordnungen überspannende Kraft, traditionale und charismatische Elemente kombinierend. Charisma spielt gerade in Demokratien eine Rolle, aber auch hier muss man spezifisch Asiatisches nicht sehen. Schauspieler wählt man daher auf den Philippinen ebenso wie in den USA, populistische Medienmogule in Thailand ebenso wie in Italien. Einige der charismatischsten und beim jeweiligen Wahlvolk erfolgreichsten politischen Führer in jüngerer Zeit sind Frauen und Ikonen von Demokratiebewegungen: Cory Aquino in den Philippinen, Aung San Suu Kyi in Burma.

Besieht man die staatsorganisatorischen Konzepte der Verfassungen Südostasiens im Detail, lassen sich die auch aus Europa und anderswo bekannten Spannweiten demokratischer Verfassungen nachweisen. Es gibt Monarchien und Republiken, präsidentielle und parlamentarische Systeme, Staaten mit und ohne verfassungsgerichtliche Kontrolle, unikamerale und bi-kamerale Parlamente. Die modernen Formen der Staatsorganisation erscheinen in vielen ihrer Varianten. Interessant sind die tendenziellen einzigartigen staatlichen Binnenstrukturen wie etwa Malaysias Modell einer föderalen Wahlmonarchie ${ }^{61}$ oder Indonesiens im Wege einer Schocktherapie implementierten Konzepts eines stark dezentralisierten Einheitsstaates ${ }^{62}$.

Für eine instruktive Analyse der Parteienlandschaft in diesen drei Staaten Andreas Ufen, Political Party and Party System Institutionalisation in Southeast Asia: A Comparison of Indonesia, the Philippines, and Thailand, GIGA Working Papers No 44, Hamburg 2007.

Max Weber, Wirtschaft und Gesellschaft, Studienausgabe Tübingen 1980, S. 122 ff.

60

Der Titel des zweiten Bandes der Memoiren Lee Kuan Yews bringt es auf den Punkt: „From Third World to First" (Singapur 2000). Selbstbewusst vermerkte Lew Kuan Yew zu den Gehältern in seiner Regierung: „If zou pay peanuts, you get monkeys.“ Immerhin gilt Singapur als einer der korruptionsfreiesten Staaten der Welt, von Transparency International weit vor Deutschland gelistet.

Zum malaysischen Bundesstaat näher Johan S. Sabaruddin, Federalism in Malaysia, in: Hill/ Menzel, (Fn. 3) Vol. III.

Zum Dezentralisierungsprozess in Indonesien s. etwa Leo Schmit, Decentralization and legal reform in Indonesia: the pendulum effect, in: Tim Lindsey (Hg.), Indonesia. Fn. 25, S. 146 ff.; Satya Arinanto, Decentralization and the Constitutional System of Government in Indonesia, in: Hill/Menzel, (Fn. 3) Vol. III; zu den hiermit verbundenen Problemen auch Gary F. Bell, The New Indonesian Laws Relating to Regional Autonomy: Good Intentions, Confusing Laws, AsianPacific Law \& Policy Journal 2 (2001), S. 1 ff. 


\section{Grundrechte}

Alle derzeit in Kraft befindlichen Verfassungen mit Ausnahme Bruneis enthalten Grundrechte ${ }^{63}$. Die Verfassungen normieren in unterschiedlicher Ausführlichkeit und Schwerpunktsetzung liberale, politische, wirtschaftliche und soziale Rechte. Autoren aus dem anglo-amerikanischen Bereich monieren häufig vorfindliche Gesetzesvorbehalte. Dem deutschen Betrachter können solche Klauseln aber im Grundsatz kaum als asiatisches Spezifikum vorkommen, finden wir sie doch auch (und im Detail nicht immer rational) auch im Grundgesetz. Ausdrückliche Vorbehalte zugunsten ,asiatischer Werte“ gibt es wie gesagt kaum, sondern im Wesentlichen solche zugunsten nationaler Sicherheit, öffentlicher Ordnung etc., ausgeprägt insbesondere in Singapur, etwas weniger offensichtlich in anderen Verfassungen der Region.

Grund- und Menschenrechte beweisen sich allerdings nicht so sehr als Verfassungstextstufen bzw. über eingegangene Vertragsverpflichtungen, sondern über ihre Umsetzung in das einfache Recht sowie die Staatspraxis - und hier stößt der südostasiatische Verfassungsstaat bislang häufig in der Tat an seine Grenzen. Unterscheiden lässt sich zwischen offiziell gewollten Beschränkungen und Problemen praktischen Nichtrespekts oder mangelnder Durchsetzungsfähigkeit. Ein Beispiel für erstere ist etwa Singapurs exzessive mandatorische Todesstrafe im Bereich von Drogendelikten ${ }^{64}$. Erhebliche Beschränkungen politisch relevanter Freiheiten wie Meinungs-, Versammlungs- und Pressefreiheit finden sich nicht nur selbstverständlich in Vietnam, Laos und Myanmar, sondern z.B. auch in Kambodscha, Malaysia und Singapur. Gerade in Singapur sind weitgehende Beschränkungen auch ausdrücklich in der Verfassung zugelassen ${ }^{65}$. Singapur hat auch, vielleicht überraschender, mit Eintritt in die Unabhängigkeit 1965 die Eigentumsfreiheit als verfassungsrechtlich garantierte Freiheit abgeschafft, um Landenteignungen vornehmen zu können, die man für die Entwicklung des territorial limitierten Stadtstaates für erforderlich hielt ${ }^{66}$. Kontroverse Beschränkungen und Tendenzen im Feld der Religionsfreiheit finden sich insbesondere in den muslimisch geprägten Staaten Malaysia ${ }^{67}$ und Indonesien ${ }^{68}$. Malaysias

Vgl. auch Randall Peerenboom / Carole J. Peterson \& Albert H.Y. Chen (Hg.), Human Rights in Asia, London \& New York 2006. Der Band enthält Beiträge zu Singapur, Malaysia, Thailand, Philippinen, Indonesien und Vietnam und diskutiert sowohl nationale als auch internationale Rechte. Zur Grundrechtssituation in Myanmar s. etwa Russell Thirgood, The State: Enemy of the People. Suppression of Human Rights in Burma, Australian Journal of Human Rights 13 (2002).

Zur Frage der Völkerrechtskonformität s. Michael Hor, The Death Penalty in Singapore and International Law, Singapore Yearbook of International Law 8 (2004), S. 105 ff. Singapurs Gerichte haben bislang weder die Verfassungswidrigkeit (Artikel 9 der Verfassung) noch Völkerrechtswidrigkeit (soweit relevant für Singapur) festgestellt (hierzu auch Li-ann Thio, Taking Rights Seriously? Human Rights Law in Singapore, in: Peerenboom u.a., (Fn. 3) S. 158 (163 f.). Vgl. Artikel 14 (2) Verfassung Singapurs. Hierzu auch Tan, Introduction, (Fn. 47) S. 177 ff.

67 Vgl. Tan, Introduction, (Fn. 3) S. 141.

Hierzu H.P. Lee, Human Rights in Malaysia, in: Peerenboom u.a., (Fn. 63) S. 191 (196 f.); ausführlich - freilich nicht mehr ganz aktuell - Poh-Ling Tan, Apostates, Deviants and Visions of 
Verfassung gewährt in Artikel 11 zwar das Recht zu Bekenntnis und Religionsausübung, spricht aber nicht von einem Recht, sich seine Religionszugehörigkeit zu wählen, insbesondere nicht, sich vom Islam abzuwenden. Hierum ranken sich derzeit zahlreiche Verfahren vor ordentlichen und Shariah-Gerichten, wobei gerade auch die Abgrenzung der Jurisdiktion dieser Gerichtszweige diskutiert wird.

Die größten Probleme in der Region bereiten im Übrigen häufig nicht „offizielle“ verfassungsrechtliche Konzepte der Beschränkung von Grundrechten, sondern der praktische Nicht-Respekt. Quer durch die Region kontrastiert weitgehende theoretische verfassungsrechtliche Gleichberechtigung von Frauen der tatsächlichen Situation im einfachen Recht und den jeweiligen Gesellschaften ${ }^{69}$. Zahlreiche Staaten sehen sich mit Vorwürfen wie extralegalen Tötungen, Landvertreibungen, Folter, unmenschlichen Haftbedingungen in Gefängnissen etc. konfrontiert. Neben staatlichen Exzessen steht staatliches Nichthandeln immer wieder im Blickpunkt. Die Kultur der Straflosigkeit, der faktischen Nichtkontrolliertheit der „rich and powerful“, wie man in Kambodscha sagt, ist nicht nur ein konkretes Grundrechtsproblem im Sinne der Nichterfüllung staatlicher Schutzpflicht, sie ist auch eines der größten Hindernisse in der Entwicklung eines allgemeinen Rechtsbewusstseins und Rechtsrespekts ${ }^{70}$. Von den überwältigenden Vollzugsproblemen im Grundrechtsschutz sind gerade auch die großen Staaten der Region betroffen, wobei neben allgemeinen und langjährigen Problemlagen immer wieder auch spezielle Problemkreise im Vordergrund stehen. Thailands Ansatz in einem sogenannten „War on Drugs“ vor einigen Jahren sowie im Rahmen von Unruhen der im Süden des Landes aufständischen Regionen sorgte für viel kritische Diskussion ${ }^{71}$. Indonesiens brutales Vorgehen in bestimmten Landesregionen zieht sich durch seine Geschichte und in West-Papua sind die Probleme nach wie vor massiv $^{72}$. Auf den Philippinen gehören die zahlreichen extralegalen Tötungen zu den aktuellen Schwerpunktthemen ${ }^{73}$.

Modernity: Contradictory Trends in Religious Freedom in Malaysia, in: Alice Tay (Hg.), East Asia - Human Rights, Nation-Building, Trade, Baden-Baden 1999, S. 108 ff..

Vgl. etwa Arskal Salim, Muslim Politics in Indonesia's De,mocratisation: The Religious Majority and the Rights of Minorities in the Post-New Order Era, in: Ross H. McLeod / Andrew MacIntyre (Hg.), Indonesia. Democracy and the Promise of Good Governance, Singapur 2007, S. 115 ff.; Denny Indrayana, Indonesian Constitutional Reform 1999-2002: Nationalism vs. Islamic State, in: The Asian Forum for Constitutional Law 2007, (Fn. 49) S. 40 ff.

Hierzu Katrin Merhof, Women's Righs in Southeast Asia's Constitutions and their implementation: The Start of a long process, in: Hill/Menzel, (Fn. 3) Vol. III. Näher Jörg Menzel, in: Hill/Menzel, (Fn. 3) Vol. II, S. 39 (63 f.). Vgl. nur Vitit Muntarbhorn, Human Rights in the era of ,Thailand Inc.“, in: Peeenboom u.a., (Fn. 63) S. 320 (325 f. mwN.).

72 Human Rights Watch, Out of Sight. Endemic Abuse and Impunity in Papua's Central Highlands, July 2007 (www.hrw.org).. 


\section{Rechtsstaat und, good governance“}

Die Praxisfragen des Grundrechtsschutzes leiten über zu allgemeinen Fragen der Rechtstaatlichkeit, die hier ebenfalls Erwähnung finden müssen, weil sie sich in den Vorgaben der ASEAN-Charter finden. Die meisten südostasiatischen Staaten nehmen zu Fragen der Rechtsstaatlichkeit Stellung ${ }^{74}$. Einige erwähnen die rule of law ausdrücklich, wie Artikel 1 Absatz 3 der indonesischen Verfassung, der in englischer Übersetzung allgemein postuliert: „The State of Indonesia shall be a state based on the rule of law. “75

Überwiegend findet sich in den von anglo-amerikanisch geprägten Autoren dominierten Diskussionen um die Rule of Law in Asien die Auffassung, dass dort ein eher formales Verständnis des Rechtsstaates überwiege und dass der Rechtsstaat eher Herrschaft legitimiere als ihr Grenzen setze. In Südostasien herrsche die Idee Rule by Law, nicht Rule of Law, heißt es zusammengefasst. Der formale Legalismus der Marcos-Diktatur wird oft angeführt, aber auch das strikt legalistische Rechtsstaatskonzept Singapurs ${ }^{76}$. Insgesamt leidet die Diskussion an der Offenheit des Begriffs der „Rule of Law“. Man wird im Übrigen vielleicht auch sagen können, dass die tendenziell unbekümmerte Ignorierung rechtlicher Prozesse das wohl noch größere Problem in Südostasiens jüngerer Geschichte war. Das extremste Beispiel findet sich in Kambodscha, wo es unter den Khmer Rouge vollständige Entrechtlichung gab. Es gab weder Gesetzgebung noch Rechtsprechung und kaum ein Jurist überlebte dieses Regime. Ein solcher Aderlass lässt sich in Generationen kaum reparieren $^{77}$.

In den Verfassungsreformdiskussionen der Region haben rule of law und „,good governance“" in jüngerer Zeit häufig eine erhebliche Rolle gespielt. Man hat sich bemüht, praktische Sicherungen für den Rechtsstaat und gegen den Machtmissbrauch einzubauen. Indonesien und Thailand haben nicht nur wie erwähnt Verfassungsgerichte, sondern, wie Vietnam, auch Verwaltungsgerichte. Die gerichtliche Überprüfung des Staatshandelns ist er-

verbatim/A-HRC8-Philippines_Advance.pdf); Human Rights Watch. Scared Silent. Impunity for Extrajudicial Killings in the Philippines, June 2007 (www.hrw.org).

74

Vgl. auch den Sammelband von Daniel Peerenboom (Hg.), Asian Discourses of Rule of Law, London \& New York 2004 (mit Beiträgen zu Vietnam, Singapur, Malaysia, Indonesien, Thailand, Philippinen).

Gerade in Indonesien ist, ein Überbleibsel niederländischer Kolonialvergangenheit, übrigens in der akademischen Debatte der Terminus Rechtsstaat gebräuchlich, ebenso wie dort lange Zeit im Kontext integralistischer Verfassungsvisionen viel von Staatsidee, Volksgeist usw. geredet wurde. $\mathrm{Zu}$ den europäischen und speziell deutschen (Savigny, Hegel) Wurzeln indonesischen Rechtsdenkens näher Peter Burns, Concepts of Law in Indonesia. The Leiden Legacy, Leiden 2004, S. 227 ff.; David Bourchier, Positivism and romanticism in Indonesian legal thought, in: Lindsey (Hg.), Indonesia, Fn. 25, S. 94 ff.

76

Zum Rule of Law-Diskurs in Singapur eingehend Li-ann Thio, Lex Rex or Rex lex? Competing Conceptions of the Rule of Law in Singapore, Pacific Basin Law Journal 20 (2002), S. 1 ff.

Näher Jörg Menzel, Kambodscha und der Kampf ums Recht. Eine Fallstudie zu Recht und Entwicklung, VRÜ 41 (2008), S. 387 ff. 
sichtlich auf dem Vormarsch. Gegenläufig zur Gesamttendenz ist allerdings Brunei, das mit der Verfassungsreform von 2004 ausdrücklich jede gerichtliche Überprüfung von Staatshandeln ausgeschlossen hat ${ }^{78}$.

Einige Verfassungen dokumentieren erheblichen Reformwillen im Feld von „good governance“. Dass zeigt sich insbesondere in der Errichtung spezieller Kommissionen und Gremien. Die aktuelle Verfassung Thailands sieht unter anderem eine Wahlkommission, einen Ombudsmann, eine Anti-Korruptionskommission, einen Rechnungshof und eine Grundrechtskommission $^{79}$ vor. Ähnliche Regelungen finden sich für die Philippinen ${ }^{80}$. In Indonesien, dessen langjähriger Staatschef Suharto mit geschätzten 15-30 Milliarden USDollar „Diebesgut“ die von Transparency International publizierte ewige Bestenliste der Staatsführer-Kleptomanen anführt ${ }^{81}$, wurde eine National Audit-Authority umfänglich in der Verfassung verankert ${ }^{82}$, und es wurden ebenfalls zahlreiche Kommissionen gegrün$\operatorname{det}^{83}$. Die 2004 eingerichtete Justizkommission geriet freilich unmittelbar in Streit mit dem korruptionsgeplagten Supreme Court, der eine Überprüfung seiner Richter durch die Kommission für unvereinbar mit der richterlichen Unabhängigkeit hielt. Das Verfassungsgericht hat diesen Standpunkt in einer kontroversen Entscheidung 2006 zwar nicht grundsätzlich geteilt, das Gesetz aber aus anderen Gründen doch für ungültig erklärt (und ungefragt gleich festgestellt, dass die Kommission jedenfalls nicht das Verfassungsgericht in den Blick nehmen könne) ${ }^{84}$.

\section{Offener Staat}

Die internationale Öffnung wird heute neben Demokratie und Grundrechten als dritte wesentliche Säule moderner Verfassungsstaatlichkeit begriffen. Die ASEAN-Charter nimmt dies insofern auf, als sie nicht nur selbst Ausdruck einer sich intensivierenden regionalen Integration ist, sondern auch ausdrücklich auf den Respekt vor dem Völkerrecht als Homogenitätskriterium hinweist ${ }^{85}$.

Section 84 C - Verfassung Bruneis; vgl. hierzu näher Tey, in: Hill/Menzel, (Fn. 3) Vol. II, S. 9 (31 f.).

79 Artikel 229 - 258 Verfassung Thailands.

80 Artikel 9: Civil Service Commission, Commission on Elections, Commission on Audit.

Transparency International, Global Corruption Report 2004 (www.transparency.org).

Artikel 23E - G Verfassung Indonesiens.

Hierzu Tim Lindsey, Legal Infrastructure and governance reform in post-crisis Asia: the case of Indonesia, in: ders. (Hg.), Law Reform in Developing and Transitional States, London \& New York 2007, S. 3 (20 ff.).

Decision No. 005/PUU-IV/2006. Hierzu näher Simon Butt, The Constitutional Court's Decision in the Dispute between the Supreme Court and the Judicial Commission: Banishing Judicial Accountability?, in: McLeod/MacIntyre, (Fn. 68) S. 178 ff.

Artikel 2 (j) der ASEAN-Charter (vgl. oben Fn. 4). Hierfür findet sich keine Entsprechung im Recht der Europäischen Union. 
Der Textbefund in den Verfassungen ist allerdings zunächst eher enttäuschend. Zunächst findet sich bislang in keiner südostasiatischen Verfassung eine spezielle Regelung zur regionalen Integration, vergleichbar den verbreiteten Europa-Klauseln in den europäischen Verfassungen. Bezüglich des Völkerrechts enthält die ausführlichsten und dezidiert völkerrechtsfreundlichen Regelungen die Verfassung Ost-Timors ${ }^{86}$. Sie sind aus Portugal abgeschrieben. In Singapur folgt die Rechtsprechung bislang durchweg englischer Doktrin, also dem dualistischen Ansatz bei völkerrechtlichen Verträgen und der grundsätzlichen Inkorporierung von Völkergewohnheitsrecht, das aber im Konfliktfall gegenüber nationalem Gesetzesrecht nachrangig ist ${ }^{87}$. Wie anderswo rankt sich in Südostasien insgesamt viel Unsicherheit um das Verhältnis von Völkerrecht und nationalem Recht ${ }^{88}$. In Kambodscha bestand zuletzt beispielsweise für einige Jahre zwischen Justiz- und Innenministerium ein Streit darüber, ob im Strafrecht die von Kambodscha ratifizierte Kinderrechtskonvention heranzuziehen sei. Der Verfassungsrat hat dies in einer zwar fast nicht begründeten, aber im Ergebnis richtigen Entscheidung im vergangenen Jahr bejaht ${ }^{89}$.

Die Staaten Südostasiens sind insgesamt eifrige Teilnehmer an den internationalen Vertragsregimen. Mit Kambodscha und Ost-Timor waren bzw. sind zwei Staaten der Region Schauplatz erheblichen Engagements der Vereinten Nationen zur (Wieder-) Herstellung funktionierender Staatlichkeit ${ }^{90}$. Zwar gibt es kaum regionales Vertragsrecht etwa in den Bereichen Menschenrechte oder Umweltschutz ${ }^{91}$, wohl aber Engagement in den globalen Mechanismen einschließlich internationaler juristischer Streitschlichtung ${ }^{92}$. Hinsicht-

Section 9, Verfassung Ost-Timors.

Näher Simon S.C. Tay, The Singapore Legal System and International Law: Influence or Interference?, in: Kevin Y.L. Tan (Hg.), The Singapore Legal System, Zweite Auflage, Singapur 1998, S. $467 \mathrm{ff}$.

Vgl. etwa Abdul Ghafur Hamid / Khin Maung Sein, Judicial Application of International Law in Malaysia: An Analysis, Asia Pacific Yearbook of International Humanitarian Law 1 (2006), S. 117 ff.; Alloysius P. Llamzon, The Generally Accepted Principles of International Law as Philippine Law, Ateneo Law Journal 47 (2002), S. 243 ff.

Näher hierzu Menzel, in: Hill/Menzel, (Fn. 3) Vol. II, S. 37 (60).

Zum Einsatz der Vereinten Nationen in Kambodscha vgl. etwa Lucy Keller, UNTAC in Cambodia - from Occupation, Civil War and Genocide to Peace, Max Planck UNYB 9 (2005), S. 127 ff.; zum Einsatz in Ost-Timor Markus Benzing, Midwifing a New State: The United Nations in East Timor, Max Planck UNYB 9 (2005), S. 295 ff. Für eine kritische Analyse des Demokratisierungserfolgs beider Einsätze vgl. Aurel Croisssant, The Perils and Promises of Democratization through United Nations Transititonal Authority. Lessons from Cambodia and East Timor, Democratization 14 (2007), S. 649 ff.

Für den Bereich der Menschenrechte s. näher Li-Ann Thio, Implementing Human Rights in ASEAN Countries: „Promises to keep and miles to go before I sleep“, Yale Human Rights and Development Law Journal 2 (1999), S. 1 ff.; für den Umweltbereich Jörg Menzel, Internationales Umweltrecht in Südostasien, AVR 2007, S. 566 (575 ff.).

Dass Asiaten aus kulturellen Gründen Gerichte nicht mögen, wird zwar häufig kolportiert, aber nicht nur stehen in Südostasien national der Ausbau und die Verbesserung gerichtlichen Rechts- 
lich der Vertragspraxis variieren die Details. Während etwa Singapur traditionell einen Ansatz verfolgt, nur nach sorgfältiger Prüfung und dann mit weitestgehendem Willen zur Vertragstreue zu partizipieren, ratifiziert Kambodscha, drastisch gesprochen, erst einmal fast alles und kümmert sich notfalls später um die Frage der möglicherweise bestehenden Verpflichtungen. Vieles hat hier wohl schlicht auch mit unterschiedlichen juristischen Expertisen und schlicht allgemein damit zu tun, wie ernst man Recht nimmt. Hinsichtlich der nationalen Vertragsschlussverfahren findet sich eine interessante Neuregelung in Thailand, wo die Regierung das Parlament zur Vermeidung von Ratifikationslageneffekten nun auch vor der Unterzeichnung von völkerrechtlichen Verträgen um Zustimmung bitten muss ${ }^{93}$. Auf den Philippinen ist anlässlich der Debatte um den Beitritt zum Internationalen Strafgerichtshof kürzlich der Versuch gescheitert, vor dem Supreme Court eine Pflicht der Regierung durchzusetzen, unterzeichnete internationale Verträge dem Parlament auch tatsächlich zur Ratifizierung vorzulegen ${ }^{94}$.

\section{Homogenität in ASEAN}

Über den Blick auf die internationale Offenheit der südostasiatischen Verfassungen gelangen wir zwanglos zurück zur ASEAN-Charter und ihren Homogenitätspostulaten. Wie bewertet man auf der Grundlage der derzeitigen Befunde in aller Vorläufigkeit die Homogenitätspostulate in ASEAN mit ihren Festlegungen auf Demokratie, rule of law, good governance und Menschenrechte?

Mit Blick auf die Regelungen der ASEAN-Charter ist es schwierig, auf Quellen zurückzugreifen, um ihre Bedeutung zu verstehen. ASEAN's Charter wurde unter weitgehendem Ausschluss der Öffentlichkeit vorbereitet ${ }^{95}$. Eine Eminent Persons Group erarbeitete das Grundkonzept. Der dann in Regierungsverhandlungen festgelegte Text der Charter blieb offiziell geheim bis unmittelbar vor der Unterzeichnung in Singapur Ende 2007. Es gibt folglich keine aussagekräftigen Erläuterungen und einstweilen soweit ersichtlich keine öffentlich zugänglichen Erklärungen, was man sich bei den einzelnen Bestimmungen gedacht hat. Wir müssen den Text also weithin „pur“ nehmen. Dabei erscheint es zunächst

schutzes weithin auf der Reformagenda, auch international sind südostasiatische Staaten zunehmend zu gerichtlicher Streitklärung bereit. Schon 1962 unterschied der Internationale Gerichtshof einen Streit zwischen Kambodscha und Thailand zur Zugehörigkeit des Tempels von Preah Vihear. In jüngster Zeit hat der Gerichtshof erneut mehrere Grenzstreitigkeiten zwischen südostasiatischen Staaten gelöst [zuletzt das Urteil des IGH v. 23.5.2008 betreffend Sovereignty over Pedra Branca/Pulau Batu Puteh, Middle Rocks (Malaysia/Singapore]). Malaysia war, um nur ein Beispiel aus dem Bereich der Welthandelsordnung zu nennen, eine treibende und besonders hartnäckige Kraft im Shrimp-Turtle-Fall. Vgl. die Nachweise bei Menzel, (Fn. 91) S. 569.

Section 190, Verfassung Thailands (2007).

94

Pimentel v. Executive Secretary, Entscheidung vom 6. July 2005; hierzu auch Harry Roque, in: Hill/Menzel, (Fn. 3) Vol. II. S. 213 (227).

Für einige Hintergrundinformation aus der Vorbereitungszeit s. Rodolfo C. Severino (Hg.), Framing the ASEAN Charter. An ISEAS Perspective, Singapur 2005. 
geboten, den Vertrag als Ganzes zu betrachten. Bemerkenswert insofern ist zunächst, dass mit der Charter ein bewusster Schritt der Verrechtlichung verbunden sein soll ${ }^{96}$. ASEAN soll aus seinem losen Aggregatzustand in einen solchen der strukturellen Verfestigung überführt werden. Von den ersten Momenten der Vorbereitung wurde dieser Gesichtspunkt betont. Die Charter schreibt in der Konsequenz nunmehr in Artikel 3 fest, dass ASEAN Rechtspersönlichkeit hat ${ }^{97}$. Die Charter ist ein echter Vertrag und nicht nur eine Deklaration wie das ursprüngliche Gründungsdokument ASEAN's aus dem Jahr 1967.

Wie „,bindend“ die für unseren Zusammenhang in Rede stehenden Bestimmungen sind, ist hiermit freilich noch nicht gesagt. Internationale Verträge mischen häufig hartes und weiches Recht und die ASEAN Charter macht wohl keine Ausnahme. Konkret mit Blick auf die hier interessierenden Homogenitätspostulate fällt zwar einerseits auf, dass die genannten Grundsätze nicht nur im Bereich der Ziele, sondern auch der Prinzipien ASEAN's auftauchen, also gewissermaßen doppelt verankert sind. Andererseits finden sich die Bestimmungen aber eben doch im eher losen Sand der einleitenden Auflistungen von Zielen und Prinzipien. Bei näherer Betrachtung erweist sich die Detailgestaltung als interessant. Während im Kontext der Ziele des Artikel 1 eine Relativierung nicht zu erkennen ist, finden sich im Rahmen der Prinzipien des Artikel 2 sehr deutlich auch die klassischen ASEANischen Konzepte der Souveränität seiner Mitgliedstaaten sowie der wechselseitigen Nichteinmischung. Man mag also argumentieren, dass im Rahmen der Zielbeschreibungen Demokratie, Menschenrechte, Rule of Law etc. unkonditioniert sind, während bei den geltenden Prinzipien wieder jedenfalls ein Konzept der Balancierung verfolgt wird. Die praktische Relevanz solcher Differenzierungen relativiert sich wohl dadurch, dass die Charter einen Sanktionsmechanismus für Vertragsverletzungen vergleichbar Artikel 7 EUV ohnehin nicht enthält. Artikel 25 thematisiert nur ankündigungsweise die künftige Etablierung von Streitentscheidungsmechanismen, die Interpretation und Anwendung der Charter betreffend.

Mit Blick auf die Beachtung von Grund- und Menschenrechten enthält die Charter in Artikel 14 zwar die Ankündigung eines regionalen Human Rights Body, was in den Medien zum Teil als Meilenstein gepriesen wurde; die Gestalt eines solches Gremiums bleibt aber ebenso offen wie die Frage, wann es sich überhaupt materialisieren wird ${ }^{98}$.

Wer nur die negative Seite der Geschichte liest, mag an den Anthropologen Clifford Geertz und seine einflussreiche Beschreibung des balinesischen Staates des 19. Jahrhun-

96

97

98

Hierzu näher Locknie Hsu, Towards an ASEAN Charter. Some Thoughts from the Legal Perspective, in: Severino, (Fn. 95) S. 45 ff.

Skeptisch bezüglich der juristischen Relevanz dieser Klausel Simon Chestermann, Does ASEAN Exist? The Association of Southast Asian Nations as an International Legal Person, Singapur 2008 (unveröffentlichtes Manuskript, zur Veröffentlichung vorgesehen für das Singapore Yearbook of International Law).

Vgl. auch Arie Afriansyah, ASEAN's Human Rights Body: New Breakthrough for Human Rights Protection in the South East Asian Region? Some Preliminary Notes from Indonesia's Perspective, Konferenzmanuskript, Singapur 2008. 
derts als Theaterstaat denken, in dem der Staatsbetrieb vor allem Inszenierung von Macht und Bedeutung ist ${ }^{99}$. Geertz' Ansatz wurde von anderen Autoren für die Gegenwart und andere Staaten in der Region fruchtbar gemacht ${ }^{100}$. Man mag versucht sein, den Gedanken auf ASEAN zu übertragen. Ist vielleicht auch ASEAN vor allem Theater? Selbst wenn man so denkt, bleibt doch interessant, dass es sich nicht um subversives Untergrundtheater handelt, in dem von Demokratie und Menschenrechten gesungen wird, sondern um offiziöses Staatstheater bzw. Staatentheater, das, selbstbestimmt, immerhin Erwartungshaltungen erzeugt.

ASEAN ist eine Regionalorganisation mit inzwischen eigener Tradition und Philosophie, die auch weiterhin starken Wert auf die Souveränität der Mitglieder und den Grundsatz der Nichteinmischung legen wird. Wenn die genannten Homogenitätsklauseln also voraussichtlich zunächst eher als weiches Recht aktualisiert werden dürften, ist dies im Vergleich zu Organisationen wie dem Europarat oder der Organisation Amerikanischer Staaten nichts Besonderes, denn auch dort werden entsprechende Bestimmungen traditionell nicht mit Konsequenz vollzogen ${ }^{101}$. Vergleiche zwischen ASEAN und der Europäischen Union, so populär sie sein mögen, sind bereits im Ansatz mit Vorsicht zu betrachten. Die neue Charter institutionalisiert keine supra-nationale Ebene, sondern baut auf der gewachsenen Struktur dieses Bundes auf. ASEAN wurde als politische Gemeinschaft gegründet, eher defensiv geeint durch die Idee des Anti-Kommunismus, nicht etwa durch „positive“ Werte wie Demokratie oder Menschenrechte, hat sich aber von dort in der 1990er Jahren zur inklusiven Regionalgemeinschaft entwickelt. Alle Staaten der Region sind heute Mitglied, mit Ausnahme des jungen Ost-Timors, dessen Antragsverfahren läuft. ASEAN hat ein für eine solche Regionalorganisation einzigartiges Spektrum an politischen Systemen unter seinem Schirm. Es hat eine erhebliche Erfolgsbilanz insofern, als es bislang keinen größeren gewaltsamen Konfliktaustrag zwischen seinen Mitgliedern gegeben hat. Man sollte solche Erfolge nicht untergewichten in einer Region, in der es durchaus viele zwischenstaatliche Konflikte gab und gibt ${ }^{102}$. Mit Blick auf fehlende Sanktionsmechanis-

99

100

101

Vgl. etwa Dolores A. Donovan, Codification in Developing Nations: Ritual and Symbol in Cambodia and Indonesia, U.C. Davis Law Review 31 (1998), S. 693 ff.

Zu den Demokratieklauseln des in der Satzung des Europarats s. Jörg Menzel, Landesverfassungsrecht, Stuttgart 2002, S. 108 f. Zu Verfassungsstaatlichkeitskriterien in der Organisation Amerikanischer Staaten auch Stephen J. Schnably, Constitutionalism and democrcatic government in the inter-American system, in: Gregory H. Fox \& Brad R. Roth (Hg.), Democratic Governance and International Law, Cambridge 2000, S. 155 ff.

102

Gerade zum Zeitpunkt der Fertigstellung dieses Beitrags gibt es freilich einmal wieder eine Konfrontation zwischen Thailand und Kambodscha über Gebietsansprüche im Bereich des Preah Vihear Tempels im Nordosten Kambodschas mit militärischem Aufmarsch auf beiden Seiten und bereits mehreren Todesfällen. ASEAN scheint hier zuletzt Bemühungen um Konfliktlösung zu entfalten (Phnom Penh Post v. 22. Oktober 2008, S. 1). 
men ist im übrigen daran zu erinnern, dass auch Artikel 7 EUV ein junges Instrument ist, Europa also trotz viel tieferer Integration lange ohne eine solche Regelung existierte ${ }^{103}$.

Im Ergebnis erscheint es angezeigt, die Homogenitätsklauseln der ASEAN-Charter juristisch nicht überzubewerten, politisch aber nicht unterzubewerten, wobei die Offenheit in der Formulierung es möglich erscheinen lässt, dass die Vertragsinterpretation über die Zeit hier auch zu anderen Ergebnissen gelangen wird. Einstweilen geben sie immerhin eine Richtung vor, reflektieren eine Tendenz und generieren Erwartungshaltungen. Man mag sie als regionales ,soft law“ qualifizieren, aber als solches sind sie nicht irrelevant. Gerade mit Blick auf Myanmar lässt sich im übrigen auch ein gewisser Wandel in der tatsächlichen Politik ASEAN's ausmachen. Chin Lim, Professor in Hong Kong, sprach bereits im vergangenen Jahr von einer neuen Politik des kollektiven Abscheus (,,collective revulsion“) ${ }^{104}$. Irgendwie erinnert das an den vor einigen Jahren unternommenen Versuch Europas, Österreichs Vertretern nicht mehr die Hand zu schütteln ${ }^{105}$. Myanmar ist einstweilen, hierüber ist man sich in der Region bewusst, Achillesverse der Gemeinschaft, auch wenn einige Regierungen vielleicht insgeheim froh sind, dass Myanmar derzeit alle Kritik auf sich zieht und Aufmerksamkeit ablenkt von Missständen in anderen Staaten.

\section{Auf dem Weg zum ,orientalischen Verfassungsstaat"?}

Nach dem Blick auf die Verfassungsentwicklungen in der Region sowie die Homogenitätsvision der ASEAN Charter sei noch einmal zusammenfassend die Frage aufgeworfen, ob es so etwas wie die Entwicklung zu einem spezifisch orientalischen Verfassungsstaat gibt, der vielleicht so etwas wie eine weichgekochte Version der orientalischen Despotie sein könnte?

Glasperlenspiele in diese Richtung könnten anknüpfen an die Asian Values Debatte ${ }^{106}$. Die Idee der Asian Values als Alternative zu westlichen Konzepten liberaler Demokratie ist unlöslich verknüpft mit einer vergangenen Generation an Staatsführern, genauer gesagt mit Singapurs Lee Kuan Yew, Malaysias Mohammed Mahathir und Indonesiens Suharto. In der Sache handelt sich um eine Mischung aus Argumenten, die in Rechtfertigung anti-

103

Umfassend Frank Schorkopf, Homogenität in der Europäischen Union, Berlin 1999; zur Geschichte der Norm knapp Matthias Ruffert, in: Christian Calliess / Matthias Ruffert (Hg.), EUV/ EGV, 2. Auflage, München 2007, Artikel 7 EUV, Rn. 1 ff.

104 C.L. Lim: From Constructive Engagement to Collective Revulsion, The Myanmar Precedent of 2007, unpublished draft (for publication in Singapore Law Review).

Hierzu etwa Frank Schorkopf, Verletzt Österreich die Homogenität in der Europäischen Union? Zur Zulässigkeit von ,,bilateralen“ Sanktionen gegen Österreich, DVB1. 2002, S. 1036 ff.

106 Vgl. aus der unüberschaubaren Literatur etwa Anthony J. Langlois, The Politics of Justice and Human Rights, Southeast Asia and Universalist Theory, Cambridge 2001; Amartya Sen, Human Rights and Asian Values, New York 1997; ders., Fn. 2, S. 231 ff.; Karen Engle, Culture and Human Rights: The Asian Values Debate in Context, International Law and Politics 32 (2000), S. 291 ff.; Simon S.C. Tay, Human Rights, Culture, and the Singapore Example, McGill Law Journal 41 (1996), S. $743 \mathrm{ff}$. 
pluralistischer Tendenzen im staatsorganisatorischen Bereich und illiberaler Ansätze im grundrechtlichen Bereich geltend gemacht wurden. Schon die Unterschiede zwischen den genannten Wortführern der Debatte zeigen aber, wie wenig es sich bei den Asian Values um ein einheitliches Konzept handelt ${ }^{107}$. Gemeinsamer Nenner war im Grunde nur ein restriktiver Ansatz im Bereich politischer Freiheit, der zum Teil mit „Development first“Argumenten und zum Teil mit einer angeblich positiveren Einstellung Asiens zu Autorität und Macht sowie einem Vorrang von Gemeinschaftsbelangen gegenüber individueller Freiheit erklärt wurde. Lew Kuan Yew's konfuzianische Asian Values Version steht insgesamt intellektuell allerdings eher mit Ostasien in Verbindung ${ }^{108}$ als mit den Konzepten seiner südostasiatischen Kollegen Suharto und Mahathir. Inzwischen sind die drei genannten Regierungschefs aus den Ämtern. In Indonesien hat ein grundsätzlicher Systemwandel stattgefunden, der Grundrechte und eine pluralistische Demokratie hervorgebracht hat, in Malaysia ist ebenfalls ein deutliches Maß demokratischer Pluralisierung zu verzeichnen und in Singapur ist die Auffassung weit verbreitet, dass ein Liberalisierungsschub einsetzen wird, sobald der langjährige Staatsführer Lew Kuan Yew endgültig das politische Parkett verlassen hat $^{109}$.

Das normative Verfassungsrecht der Region stützt Theorien einer spezifisch südostasiatischen Version des Verfassungsstaates ohnehin kaum. Südostasiens Verfassungen sind nicht traditional in ihrer Anlage und es gibt kein auch nur ansatzweise einheitliches Muster anti-liberaler Herrschaft. Anders als im benachbarten Südpazifik, wo Verfassungen häufig in normativ relevanter Weise auf die Traditionen des Landes Bezug nehmen und zum Teil sogar Verfassungsorgane schaffen, die in Tradition begründet sind ${ }^{110}$, geben sich Südostasiens Verfassungen weithin modern ${ }^{111}$.

Unbestritten ist freilich, dass es auch heute noch einen gewissen Hang zum Autoritären im Staats- und Rechtsleben der Region gibt. Dies ist evident für die Verfassungsordnungen in Brunei, Myanmar, Vietnam und Laos, unter Einbeziehung von einfachem Recht und Staatspraxis aber auch in weiteren Staaten der Region. Freedom House stuft in der letzten

107

Instruktive Darstellung der verschiedenen Ansätze in Malaysia, Indonesien und Singapur bei Langlois (Fn. 105), S. 12 ff.

Es erscheint bemerkenswert, dass die sonstigen mehr oder weniger konfuzianischen Staaten Ostasiens, die zum Teil argumentativ dafür bemüht wurden, dass autoritäre Strukturen regional adäquat seien, inzwischen allgemein als freiheitlich eingestuft sind. Immerhin Japan wie Deutschland nach dem zweiten Weltkrieg erst demokratisiert, um sodann sein Wirtschaftswunder zu erfahren. Auch für Südkorea und Taiwan bleibt unbelegt, dass der Aufschwung nicht mindestens gleich erfolgreich gewesen wäre, hätte Demokratisierung früher eingesetzt.

Lee Kuan Yew, derzeit 85 Jahre alt, ist seit 2004 „Minister Mentor“ im Kabinett von Premierminister Lee Hsien Loong (seines Sohnes).

110 Vgl. näher Jörg Menzel, Der Südpazifik: Inselwelten zwischen verfassungsrechtlicher Modernität und gesellschaftlicher Tradition, Verfassung und Recht in Übersee 1997, S. 573 ff.

111

Gelegentliche Traditionsklauseln schließt das nicht aus, vgl. etwa Artikel 69 der Verfassung Kambodschas, der die nationale Kultur, Sprache sowie archäologische Schätze unter Schutz stellt. 
Ausgabe seiner Freiheitsweltkarte nur Indonesien als frei ein, alle anderen Staaten der Region als teilweise frei oder unfrei ${ }^{112}$. Man kann über die Details solcher Messungen streiten, aber es liegt auf der Hand, dass ASEAN's Ziele und Prinzipien vor dem Hintergrund des tatsächlichen Stands der Dinge in gewisser Weise visionär sind. Dabei gibt es keinen Anhaltspunkt für eine Interpretation der Begriffe in einem spezifisch asiatischen Sinne dahingehend, dass all die derzeitigen Realitäten als unproblematisch anzusehen wären. ASEAN's Amtssprache ist Englisch und die Begriffe stehen dort ohne interpretatorische Erklärungen. Das ist eine erhebliche Entwicklung fünfzehn Jahre nach der Bangkok Deklaration über Menschenrechte ${ }^{113}$, die gewissermaßen den Zenith des Asian Values Diskurses markiert.

Gerade dem visionären Element der Charter gebührt Respekt, denn es ist einfach, nur zu normieren, was man schon für Sachstand hält. Wenn ASEAN nun offensiv und positiv über Demokratie und Menschenrechte spricht, ohne im Stile der Bangkok Deklaration Referenzen zu machen, dass es sich jeweils um ein originär asiatisches Verständnis handeln würde, erscheint dies als weiterer Beleg dafür, dass es sich jedenfalls im Kern eben doch nicht nur um „,westliche“ Werte handelt. Dem Gedanken der Universalität der Menschenrechte wird hier ebenso zugearbeitet wie einem (in aller Vorsicht zu definierenden) völkerrechtlichen Anspruch auf Demokratie ${ }^{114}$. Die Begriffe sind nach wie vor offen für regionale Akzentuierungen, aber eine Perspektive spezifisch orientalischer Verfassungsstaatlichkeit scheint jedenfalls derzeit und im Gegensatz zu den 1990er Jahren nicht mehr auf der offiziellen Agenda in Südostasien zu stehen. Südostasien befindet sich damit auf einem Weg, den die entwickelteren ostasiatischen Staaten (Japan, Süd-Korea, Taiwan) bereits hinter sich gebracht haben ${ }^{115}$.

\section{Schluss}

Der ungezähmte Leviathan stand unter Naturschutz in Südostasien für einige Zeit, und oft wurde er gefüttert aus Washington, Moskau, Peking etc. Nun ist er ein gejagtes Ungeheuer. Südostasien ist und bleibt einstweilen zwar eine Region voller politischer und verfassungsrechtlicher Vielfalt. Normativ betrachtet wird das selbstbestimmte regionale Homogeni-

Abrufbar unter www.freedomhouse.org. Zum Vergleich: Alle EU-Mitglieder sind bei Freedom House als frei eingestuft.

113 Final Declaration of the Regional Meeting of Asia of the World Conference on Human Rights vom 7. April 1993 (P 8, A/CONF.157/ASRM/8-A/CONF.157/PC/59).

Hierzu auch Thilo Rensmann, Modern Constitutionalism between Regional and Universal Values. Constitutionalism in Southeast Asia, in: Hill/Menzel, (Fn. 3) Vol. III. Zum völkerrechtlichen Anspruch auf Demokratie allgemein grundlegend Thoma M. Franck, The Emerging Right to Democratic Governance, AJIL 86 (1992), S. 46 ff. sowie den Sammelband von Fox \& Roth, (Fn. 101).

Für eine Kritik der Asian Values Doktrin als Gegenargument zum modernen Gedanken des Verfassungsstaates auch Tatsuno Inoue, The Necessity of Difficulty of Constitutionalism in the Contemporary World, in: The Asian Forum for Constitutional Law 2007 (Fn. 49), S. 5 ff. 
tätsmodell mit der ASEAN-Charter nun aber dasjenige des modernen Verfassungsstaates, basierend auf Demokratie, Menschenrechten, Rechtsstaat und internationaler Offenheit. Dass es einen Sog in Richtung dieser Werte auch im Staatsleben der Region gibt, ist nicht zu übersehen, betrachtet man die Region als Ganzes und mit einem gewissen historischen Horizont. Bruce Ackerman argumentiert in seinem 1997 erschienenen Beitrag ,The Rise of World Constitutionalism“, man solle sechzig Jahre zurückblicken, um sich den Fortschritt zu vergegenwärtigen ${ }^{116}$. In Südostasien reicht 2008 eine Rückblende um gut zwei Jahrzehnte, und die Reichweite des Wandels wird unmittelbar deutlich. Im Jahr 1985 waren Indonesien, die Philippinen und Thailand rechte Diktaturen, Vietnam, Laos und Kambodscha linke Diktaturen. Mit Ausnahme von Vietnam und Laos haben heute alle dieser Staaten demokratisch-menschenrechtlich ausgerichtete Verfassungen, und überall hat sich die Situation wohl jedenfalls verbessert. Demokratie, Grundrechtsschutz und Rechtsstaat sind nach wie vor keine Selbstverständlichkeiten in der Region, aber die Situation ist (gerade in den meisten der größeren Staaten) besser als vor zwei Jahrzehnten, und die Perspektiven sind vielleicht positiver, als häufig angenommen wird ${ }^{117}$.

Europäische Bilder über die Ferne sind oft eher faktenfrei als imaginative Gegenbilder des Eigenen entstanden. Das gilt für die ,orientalische Despotie“ ebenso wie für die Rousseau'sche Vision vom „edlen Wilden“ im Südpazifik ${ }^{118}$. Akademische Vernachlässigung unterstützt das Fortleben fehlerhafter Vorstellungen. Andrew Harding beschreibt in einem Beitrag aus dem Jahr 2002 nicht nur den Grad der Vernachlässigung dieser sich dynamisch entwickelnden Region. Er weist auch ironisch auf den Versuch Ugo Mattei's hin, eine Taxonomie der Rechtsordnungen Südostasiens in einer einzigen Fußnote irgendwo zwischen politisch und traditional vorzunehmen ${ }^{119}$. In Wirklichkeit sind Recht und Verfassungsrecht in Südostasien gleichermaßen divers. Wie hierzulande hängen Recht und Verfassung dabei offensichtlich zusammen. Diskussionen über Recht (wie auch über Politik) drehen sich nicht zuletzt um die Verwirklichung verfassungsrechtlicher Verheißungen und die Verfassungsmäßigkeit des Staatshandelns. Nicht nur ein vermehrtes akademisches

116

117

Ackerman (Fn. 23), S. 771.

Für eine tendenziell positive Einschätzung der Verfassungsentwicklung in Asien auch Tom Ginsburg, Lessons for Democratic Transitions: Case Studies from Asia, Illinois Public Law and Legal Theory Research Papers Series (No. 07-08), 2007; allzu optimistisch freilich Michael Vatikiotis, Southeast Asia in 2005. Strength in the Face of Adversity, in: Southeast Asian Affairs 2006, S. 3 (14), nach dem nun mit ein oder zwei Ausnahmen alle Staaten der Region "firmly democratic" seien. Sehr viel skeptischer im Übrigen ein Jahr später die Einschätzung in dem entsprechenden Einführungsbeitrag von Donald E. Weatherbee, Southeast Asia in 2006, Déjà vu all over again, Southeast Asian Affairs 2007, S. 3 (27): „Democracy is under fire“.

118 Vgl. hierzu die Anthologie von Richard Lawdson (Hg.), Strangers in the South Seas. The Idea of 119 the Pacific in Western Thought, Honolulu 2006 (mit Beiträgen zum "noble savage" S. 64-109).

Harding (Fn. 12), S. 264 f. Die Kritik bezieht sich auf Ugo Mattei, Three Patterns of Law: Taxonomy and Change in the World's Legal Systems, American Journal of Comparative Law 45 (1997), S. 1 (Fn. 135). Vgl. auch schon oben Fn. 12. 
Interesse in Deutschland an solchen Prozessen wäre erfreulich, sondern auch ein intensivierter Dialog mit der zum Teil noch im Aufkeimen befindlichen Verfassungsrechtswissenschaft in Südostasien.

Überblickt man die zurückliegenden gut zwanzig Jahre, offenbaren sich wie gesagt Dynamik und Umfang des Wandels, der zwar nicht linear ist und auch scharfe kurzfristige Konjunkturkurven aufweist, aber doch insgesamt eine Stärkung verfassungsstaatlicher, demokratischer und menschenrechtlicher Konzepte mit sich gebracht hat. Insofern beschreiben die neuen verfassungsrechtlichen Homogenitätsstandards der neuen ASEAN Charter zwar nicht den Stand der Dinge, sie reflektieren aber eine Tendenz. Dass sich solche Konzepte immer wieder gegen autoritäre und illiberale Angriffe behaupten müssen, ist mitnichten ein spezifisch südostasiatisches Phänomen, wo ein Ende der Geschichte ebenso wenig in Sicht ist wie anderswo. 